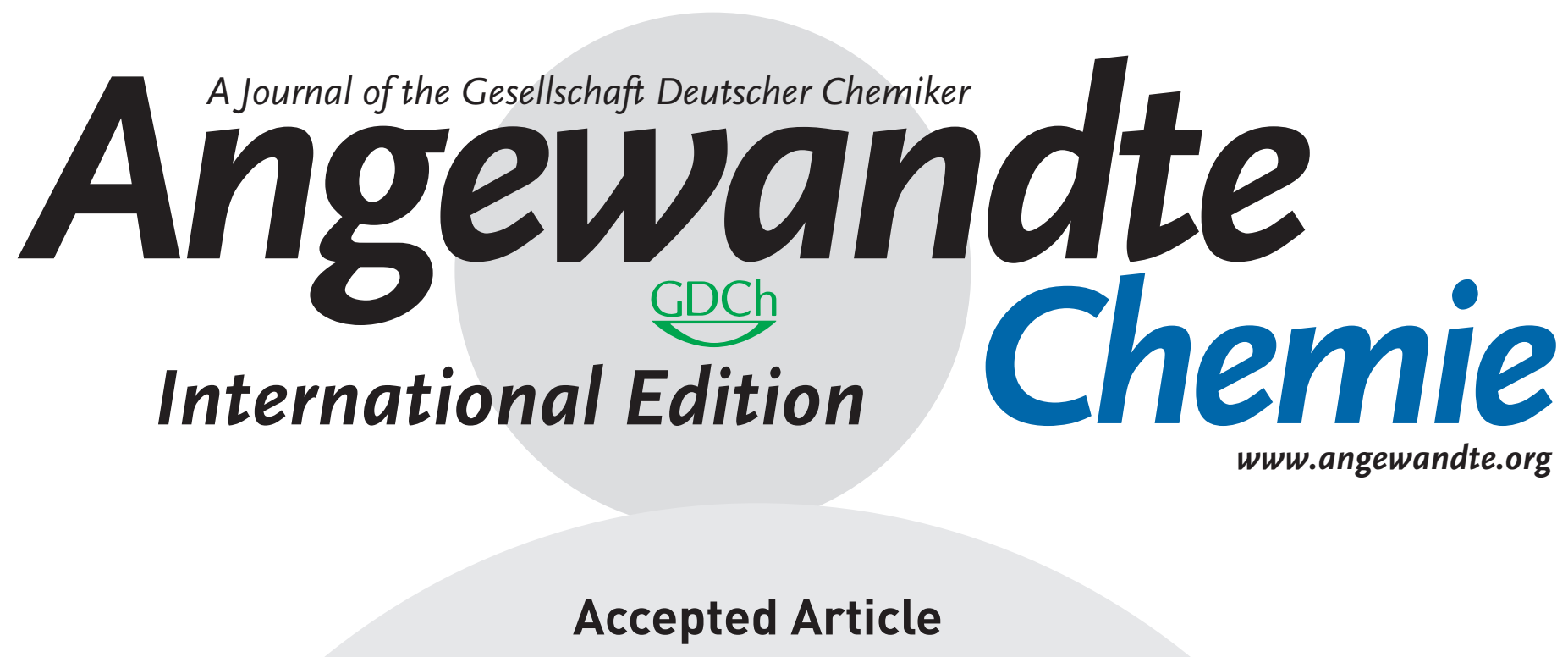

Title: Asymmetric Alkylation of Ketones Catalyzed by Engineered TrpB

Authors: Ella J. Watkins-Dulaney, Noah P. Dunham, Sabine Straathof, Soma Turi, Frances H. Arnold, and Andrew Richard Buller

This manuscript has been accepted after peer review and appears as an Accepted Article online prior to editing, proofing, and formal publication of the final Version of Record (VoR). This work is currently citable by using the Digital Object Identifier (DOI) given below. The VoR will be published online in Early View as soon as possible and may be different to this Accepted Article as a result of editing. Readers should obtain the VoR from the journal website shown below when it is published to ensure accuracy of information. The authors are responsible for the content of this Accepted Article.

To be cited as: Angew. Chem. Int. Ed. 10.1002/anie.202106938

Link to VoR: https://doi.org/10.1002/anie.202106938 
WILEY-VCH

\title{
Asymmetric Alkylation of Ketones Catalyzed by Engineered TrpB
}

\author{
Ella J. Watkins-Dulaney, ${ }^{[a]}$ Noah P. Dunham, ${ }^{[b]}$ Sabine Straathof, ${ }^{[b]}$ Soma Turi, ${ }^{[b]}$ Frances H. Arnold ${ }^{*[a, b]}$, \\ Andrew R. Buller*[c], \\ [a] E. J. Watkins-Dulaney, Prof F. H. Arnold \\ Division of Biology and Biological Engineering \\ California Institute of Technology, MC 210-41 \\ 1200 E. California Boulevard, Pasadena, CA, 91125 (USA) \\ E-mail: frances@cheme.caltech.edu \\ [b] Dr. N. P. Dunham, S. Straathof, S. Turi, Prof F. Arnold \\ Division of Chemistry and Chemical Engineering \\ California Institute of Technology, MC 210-41 \\ 1200 E. California Boulevard, Pasadena, CA, 91125 (USA) \\ E-mail: frances@cheme.caltech.edu \\ [c] Prof. A.R. Buller \\ Department of Chemistry \\ University of Wisconsin - Madison \\ 1101 University Ave, Madison, WI, 53706, USA \\ Supporting information for this article is given via a link at the end of the document.
}

\begin{abstract}
The $\beta$-subunit of tryptophan synthase $(\operatorname{TrpB})$ catalyzes a PLP-mediated $\beta$-substitution reaction between indole and serine to form L-Trp. A succession of TrpB protein engineering campaigns to expand the enzyme's nucleophile substrate range has enabled the biocatalytic production of diverse non-canonical amino acids (ncAAs). Here, we show that ketone-derived enolates can serve as nucleophiles in the $\operatorname{TrpB}$ reaction to achieve the asymmetric alkylation of ketones, an outstanding challenge in synthetic chemistry. We engineered TrpB by directed evolution to catalyze the asymmetric alkylation of propiophenone and 2-fluoroacetophenone with a high degree of selectivity. In reactions with propiophenone, preference for the opposite product diastereomer emerges over the course of evolution, demonstrating that full control over the stereochemistry at the new chiral center can be achieved. The addition of this new reaction to the TrpB platform is a crucial first step toward the development of efficient methods to synthesize non-canonical prolines and other chirally dense nitrogen heterocycles.
\end{abstract}

The formation of carbon-carbon $(\mathrm{C}-\mathrm{C})$ bonds in a controllable and selective manner is central to the construction of the diverse organic scaffolds upon which biological systems depend. While the fundamental challenges of $\mathrm{C}-\mathrm{C}$ bond formation are constant, the ensuant solutions put forth by Nature and the synthetic chemist are distinct yet complementary. Nature has met these challenges with a relatively modest set of $\mathrm{C}-\mathrm{C}$ bond-forming enzymes that operate under mild conditions and exhibit astonishing control over chemo-, regio-, and stereo-selectivities..$^{[1,2]}$ By contrast, chemists have accumulated an arsenal of creative methods, many of which boast impressively large scopes of useful substrates. To overcome deficiencies in selectivity, however, chemists often employ preciousmetal catalysts and complex ligands, and rely on addition and removal of protecting groups, which can quickly result in inefficient, multistep syntheses. Furthermore, most of these reactions are not compatible with aqueous solutions, and instead require harsh conditions and environmentally hostile solvents. ${ }^{[3]}$ New catalytic methods for $\mathrm{C}-\mathrm{C}$ bond formation that draw from the vast array of nucleophilic substrates in the synthetic literature while leveraging the renowned selectivity of enzymes would be highly useful.
The tryptophan synthase $\beta$-subunit (TrpB) is a pyridoxal phosphate (PLP)-dependent, $\mathrm{C}-\mathrm{C}$ bond-forming enzyme that catalyzes the last stage in tryptophan biosynthesis. Natively, TrpB is bound to, and allosterically activated by, the tryptophan synthase $\alpha$-subunit (TrpA), which cleaves indole-3-glycerol phosphate into indole and glyceraldehyde-3-phosphate. ${ }^{[4]}$ Indole diffuses along a $25 \AA$ tunnel from TrpA to TrpB, where the PLP cofactor mediates $\mathrm{C}-\mathrm{C}$ bond formation between $\mathrm{C}_{3}$ of free indole (in preference to $N_{1}$ ) and an electrophilic serine-derived aminoacrylate species $(E(A-A)$, Figure $1 A)$. A recent engineering study demonstrated that activity lost by removal of TrpA could be recovered by directed evolution, leaving the stand-alone TrpB subunit as a simpler, more readily programmable platform for the synthesis of non-canonical amino acids (ncAAs). ${ }^{[5]}$

The electrophilic amino-acrylate intermediate of the native TrpS complex can react with nucleophilic species other than indole: the complex can accept molecules such as indoline, aniline, benzenethiol, and benzeneselenol to form new $\mathrm{C}-\mathrm{N},{ }^{[5-10]}$ $\mathrm{C}-\mathrm{S},{ }^{[11]}$ and $\mathrm{C}-\mathrm{Se}$ bonds, ${ }^{[11]}$ respectively (Figure 1B). By engineering the stand-alone TrpB (vide supra), Buller, Arnold, and coworkers systematically broadened the scope of ncAAs that are accessible using various nucleophiles, including an assortment of substituted indoles, ${ }^{[5,9,12,13]}$ azulene, ${ }^{[14]}$ nitroalkanes, ${ }^{[15]}$ and oxindoles $^{[16]}$ (Figure 1B). Impressively, the TrpB variant engineered to utilize 3 -substituted oxindoles facilitates the formation of quaternary $\mathrm{C}-\mathrm{C}$ bonds in preference to $\mathrm{C}-\mathrm{N}$ bond formation in a powerful demonstration of how enzyme selectivity can be leveraged for the construction of $\mathrm{C}-\mathrm{C}$ bonds that are otherwise challenging to make. ${ }^{[16]}$

In this study, we investigated the capacity of ketone-derived enolates to serve as nucleophiles in TrpB-catalyzed reactions (Figure 1C). While these compounds are one of the most wellresearched carbon-based nucleophiles in the synthetic literature, there are still no general methods for their direct asymmetric $\alpha$ alkylation. ${ }^{[3]}$ Enzymatic reactions with enolates are relatively rare, and to our knowledge there are no examples engineering enzymes to accept enolates if they are not already the native substrate. Nevertheless, we hypothesized TrpB might be able to accomplish this feat, and that realizing this chemistry may inspire 
others to investigate further chemoenzymatic reactions with enolates. At the outset, we considered four main obstacles to achieving selective alkylation of ketones: (1) ketone tautomerization can result in multiple enolate stereo- and regioisomers, the latter stemming from asymmetrical ketones with multiple deprotonation sites; (2) the facial approach of the enolate to the electrophile dictates the Ca stereochemistry and therefore must be precisely controlled to achieve stereoselectivity; (3) the substituted ketone product may also tautomerize and racemize the newly set chiral center; (4) enols are thermodynamically disfavored in aqueous conditions, so the population of reactive nucleophile available in solution would be very small. We reasoned that if $\operatorname{TrpB}$ could accept enolates as nucleophiles, the selectivity imparted by the enzyme active site could conquer the first two obstacles.

A
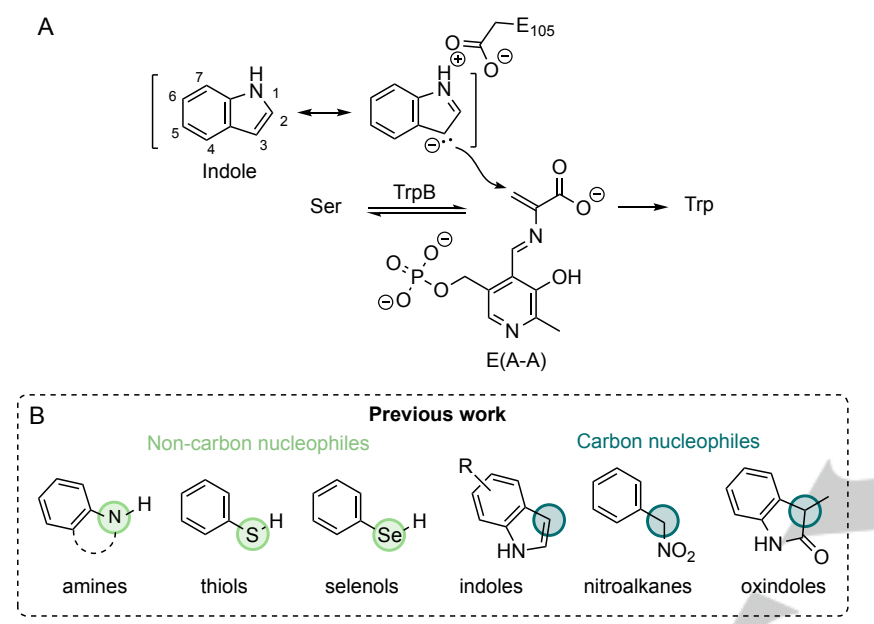
Tautomerization

Figure 1.) The $C-C$ bond-forming step in TrpB-catalyzed reactions: nucleophilic addition of $\mathrm{C}_{3}$ of indole to the amino acrylate $(\mathrm{E}(\mathrm{A}-\mathrm{A}))$. B) Nucleophiles previously shown to be used by $\operatorname{TrpS} / \operatorname{TrpB}$. C) Enolate nucleophiles, supplied to the reaction as their ketone tautomer, can be used by TrpB to form linear ncAAs that undergo spontaneous cyclization.

We began by screening a panel of purified TrpB enzymes from previous evolution projects for activity with serine and propiophenone. We selected propiophenone because it absorbs light in the ultraviolet (UV) region, bears a bulky phenyl substituent that resembles other non-indole nucleophiles that react with TrpB, and has been highlighted as a ketone for which no direct asymmetric alkylation methods have been reported. ${ }^{[3]} \mathrm{A}$ Thermotoga maritima TrpB (TmTrpB) variant previously engineered to synthesize 4-cyanotryptophan ${ }^{[12]}$-Tm9D8*- displayed trace activity with propiophenone (30 TTN, Figure 2A) to form a mixture of cyclized imine isomers as detected by HPLCMS (vide infra).

To improve this novel enzymatic reactivity, we initiated a directed evolution campaign using a combination of sitesaturation and random mutagenesis and screened the mutant libraries for increases in product formation, remaining agnostic as to whether beneficial mutations resulted from enhanced enzymatic activity or expression (See supporting information for further detail). We screened lysate reactions at $37{ }^{\circ} \mathrm{C}$ during the first round of engineering, as this temperature elicited the highest activity of the Tm9D8* variant in previous reactions with 4-cyanotryptophan. ${ }^{[12]}$ Optimizing the reaction conditions, we found product formation with the propiophenone substrate reached a maximum at $55^{\circ} \mathrm{C}$, and this temperature was used for subsequent rounds of directed evolution. Three rounds consecutively accrued the mutations F184H (TmE1, 26 TTN), I174T (TmE2, 28 TTN), and F41Y (TmE3, 39 TTN; Figure 2A).

Constrained to product detection by HPLC-MS, limited analytic screening capacity quickly became an obstacle to discovering improved variants. Thus, we explored screening by Multiple Injections in a Single Run (MISER), a flow-injection analysis method in which each sample in a 96-well plate is injected sequentially, passed through a short guard column, and analyzed by the mass spectrometer. ${ }^{[17]}$ This strategy dramatically improved screening throughput, decreasing the time per 96-well plate from 5 hours to 35 minutes. With MISER, we screened a larger error-prone PCR (epPCR) random mutagenesis library and found an improved variant bearing four additional mutations: K39I, E40G, P54Q, and I195T (TmE4, 53 TTN; Figure 2A). During this round of engineering, however, the poor signal-to-noise ratio of the MISER screen resulted in a number of false positives that impeded the overall workflow. Therefore, we decided to examine the substrate scope of TmE4 with the prospect of uncovering a new substrate that could be detected by MISER more reliably. To our delight, TmE4 exhibited much higher activity with 2fluoroacetophenone (90 TTN, Figure 2B), which is consistent with the expected activating effect of the fluorine on the $\mathrm{Ca}-\mathrm{H}$ bond.

For the remainder of the evolution, we screened for activity with serine and 2-fluoroacetophenone using the MISER analysis method. In the next two rounds of engineering, several variants that yielded improvements were identified (TmE5, TmE6.3, and TmE6.6; Figure 2B and Supporting Information). With a considerable number of mutations now observed throughout the lineage, we generated a recombination library using staggered extension process (StEP) PCR, ${ }^{[18]}$ which allowed each residue of interest to either retain its mutation or revert back to wild type. Of the 600 colonies screened, the best variant displayed a further 2fold increase in activity over the best round 6 variant, TmE6.3, by recombining mutations $1166 \mathrm{~V}$ and S228G (TmE7, 700 TTN; Figure 2B). 
A

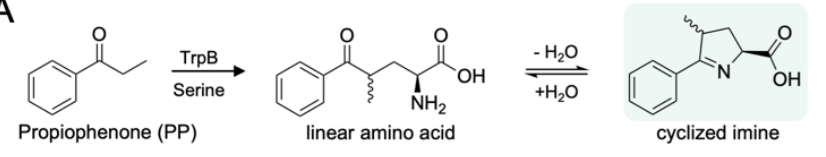
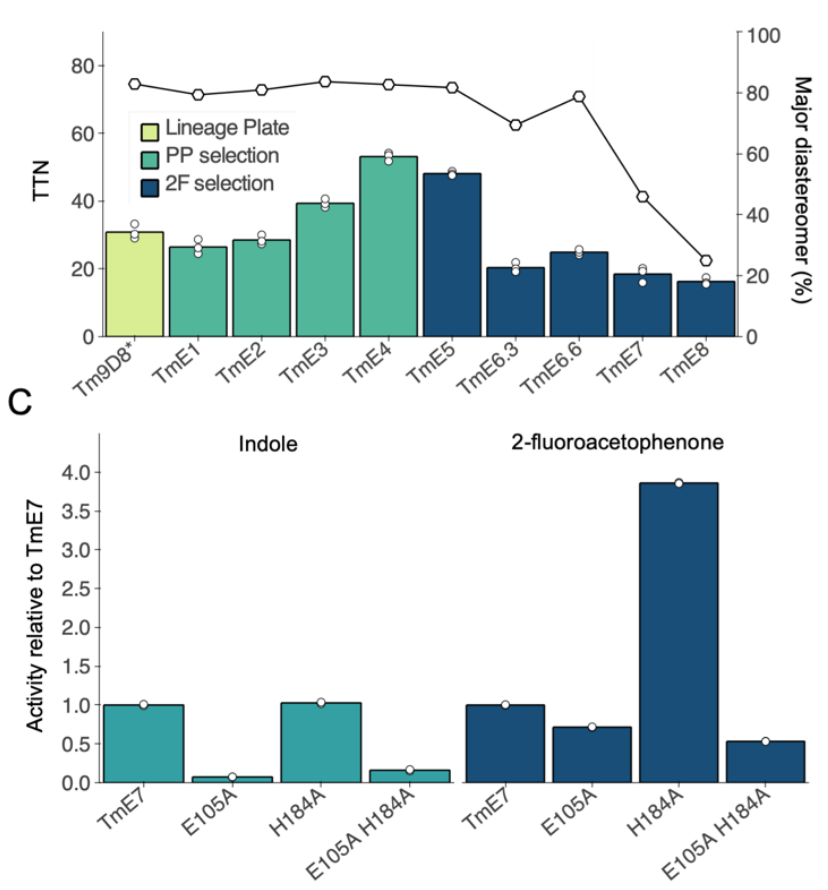

B

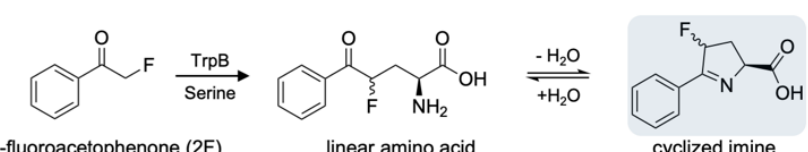

2-fluoroacetophenone (2F) linear amino acid cyclized imine
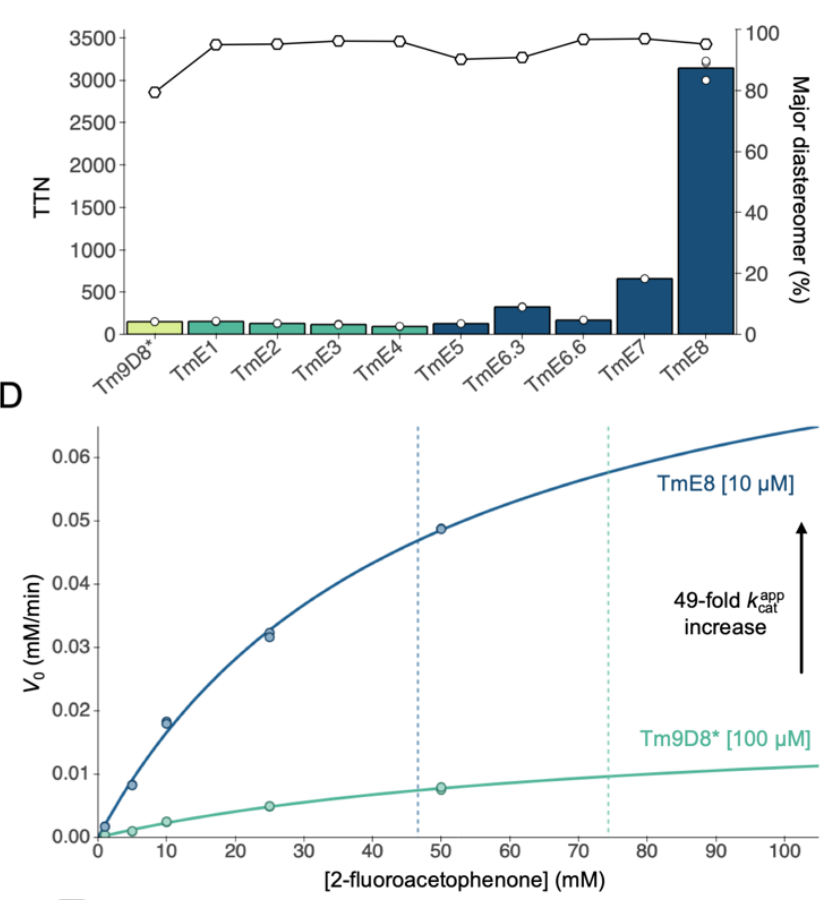

Figure 2. A) Activity of variants in the TrpB evolutionary lineage with propiophenone (PP) and serine. Bars represent the average of two or three replicates shown as individual points. Colors refer to the substrate used for screening. The diastereoselectivity, shown as individual points, for the cyclized imine product was measured for each variant. B) Activity of variants in the TrpB evolutionary lineage with 2-fluoroacetophenone (2F) and serine. Bars represent the average of two or three replicates shown as individual points. Colors refer to the substrate used for screening, as indicated in panel A. The diastereoselectivity, shown as individual points, for the cyclized imine product was measured for each variant. C) Activities of alanine mutants with indole and 2-fluoroacetophenone, relative to TmE7. D) Michaelis-Menten kinetics of Tm9D8* (teal) and TmE8 (dark blue). Dotted lines represent the $K_{\mathrm{M}}^{\text {app }}$. Reaction conditions for Figure 2 can be found in SI Section 2.8 and 2.9 .

Because enolates are structurally and electronically dissimilar to the reactive indole enamine-like species, we investigated the roles of active-site residues E105 and $\mathrm{H} 184$ by introducing alanine mutations at each site and measuring changes in activity for both indole and 2-fluoroacetophenone. E105 facilitates nucleophilic attack on the amino acrylate by stabilizing the accumulation of positive charge on the pyrrole ring of indole (Figure 1A); the E105G mutation in TmTrpB was shown to be deleterious to activity with indole and several analogs. ${ }^{[14]}$ Previous studies have shown that mutations to $\mathrm{H} 184$, which is spatially adjacent to E105, also strongly affect activity, ${ }^{[12,15]}$ although the specific role of histidine is less clear. The E105A mutation reduced the indole activity of TmE7 unsurprisingly by $93 \%$, but only decreased the 2-fluoroacetophenone activity by $30 \%$ (Figure 2C). While the H184A mutation had no effect on indole activity, we were pleasantly surprised to find that it improved 2-fluoroacetophenone activity 3.8-fold. TrpB TmE7 with the H184A mutation (TmE8, 3140 TTN, $86 \%$ yield by HPLC; Figure 2B) was used for further studies of this new reactivity.

Using the best variant for each respective substrate, we isolated the products from enzymatic reactions for NMR characterization, as reported syntheses for making the authentic standards were absent from the literature. The enzymatic products of the reactions with propiophenone and 2- fluoroacetophenone were each identified to be a mixture of two imine isomers. Reapplication of the propiophenone-derived imine product mixture as an authentic standard showed that directed evolution had a strong influence on the diastereomeric outcome (Figure 2A). Although we had not screened specifically for diastereoselectivity, the preference for a single diastereomer was inverted between Tm9D8* and TmE8 (82:18 to 24:76 diastereomeric ratio, d.r., Figure 2A), demonstrating that stereochemistry at the newly formed chiral center can be tuned with this platform. In reactions with 2-fluoroacetophenone, the enzymes exhibited an even higher degree of selectivity, with the final variant, TmE8, boasting a d.r. of 95:5 (Figure 2B). However, we observed an erosion in selectivity at prolonged reaction times (Figure S2), suggesting the newly formed chiral center racemizes through tautomerization in solution, as discussed above. In principle, subsequent reduction of the product would fix the stereochemistry, and indeed we observed the expected shifts in product mass by HPLC-MS upon treatment with sodium cyanoborohydride (Figure S3), but the resulting mixture of diastereomers rendered rigorous product characterization problematic. Leveraging the selectivity of a suitable reductase in an enzymatic cascade may be the best solution to this problem (vide infra). 
A
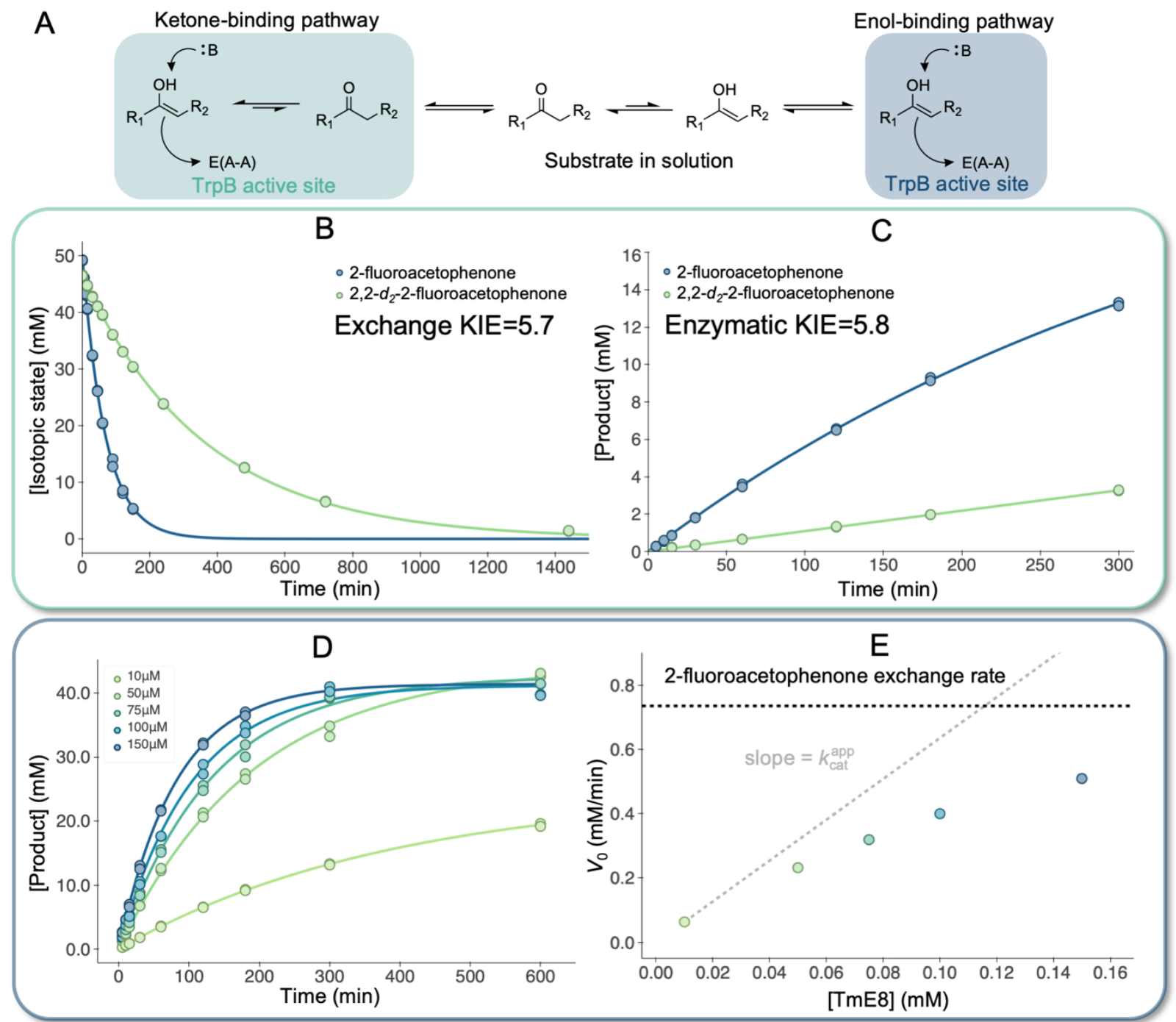

Figure 3 A) Possible pathways to enolate formation. B) Exchange curves of 2-fluoroacetophenone and 2,2- $d_{2}$-2-fluoroacetophenone in deuterated buffer and unlabeled buffer, respectively $\mathrm{C}) \mathrm{TmE} 8(10 \mu \mathrm{M})$ progress curves with 2-fluoroacetophenone and 2,2- $d_{2}$-2-fluoroacetophenone at $50 \mathrm{mM}$ substrate loading. D) TmE8 progress curves at varying [enzyme] with $50 \mathrm{mM}$ 2-fluoroacetophenone. E) Initial velocty of product formation extracted from the progress curves in $D$ represented as a function of [TmE8]. Details of nonlinear regression analyses can be found in SI Section 2.8 and 2.10.

To gain insight into the mechanism of this new transformation, we first explored the kinetics of product formation in reactions with 2-fluoroacetophenone catalyzed by TM9D8* and TmE8 (Figure 2D). TmE8 exhibited a 49-fold increase in apparent turnover number $\left(k_{\text {cat }}^{\text {app }}\right)$ compared to Tm9D8* $\left(9.4\right.$ min $^{-1}$ and 0.19 $\mathrm{min}^{-1}$, respectively), which is likely the principal factor contributing to the 20-fold increase in TTN observed across the evolutionary lineage. The change in the apparent $K_{\mathrm{M}}\left(K_{\mathrm{M}}^{\mathrm{app}}\right)$ was modest $(74$ $\mathrm{mM}$ and $47 \mathrm{mM}$ for Tm9D8* and TmE8, respectively). More accurate measurements were limited by the poor solubility of 2fluoroacetophenone beyond $100 \mathrm{mM}$. Nevertheless, the dramatic improvement in $k_{\mathrm{cat}}^{\mathrm{app}}$ without a significant change in the strikingly high $K_{\mathrm{M}}^{\text {app }}$ values prompted us to investigate whether the enzyme was binding the ketone and facilitating its tautomerization in the active site or simply reacting with the small population of the enol tautomer present in solution (Figure $\mathbf{3 A}$ ).

We measured the rate of deuterium exchange into 2fluoroacetophenone from deuterated buffer in the absence of enzyme (Figure 3B). Under these conditions, the rate of uncatalyzed tautomerization is an order of magnitude faster than the enzymatic reaction (Figure $\mathbf{3 C}$ ). We measured the rate of protium exchange into 2,2- $d_{2}$-2-fluoroacetophenone in unlabeled buffer, from which we calculated a kinetic isotope effect (KIE) of 5.7 for uncatalyzed deprotonation of the starting material (Figure 3B). We note that the background rate of enolization is calculated by measuring the formation of isotopically labeled ketone resulting from two tautomerization events and is a conservative approximation of the in-solution tautomerization rate, as a singlestep conversion to the enol would be marginally faster. To learn more about tautomerization in the enzyme active site, we extracted the initial velocity $\left(V_{0}\right)$ of product formation from the progress curves of TmE8 with the unlabeled 2fluoroacetophenone substrate and 2,2- $d_{2}$-2-fluoroacetophenone and determined a KIE of 5.8 (Figure $3 \mathrm{C}$ ). The remarkable similarity of these two KIEs suggests that the KIE measured in the enzymatic reaction may also originate from in-solution tautomerization of the ketone. We therefore considered a second scenario wherein TmE8 binds the enol tautomer directly (Figure 
3A, Enol-binding pathway). Based on known equilibrium constants of acetophenone tautomerization, ${ }^{[19,20]}$ we estimate that the 2-fluoroacetophenone-derived enol tautomer is present at low $\mu \mathrm{M}$ concentrations for these experiments. Under these conditions, approximately a single turnover of enzyme (present at $10 \mu \mathrm{M}$ ) would deplete the starting material entirely, obliging the system to then wait for tautomerization to deliver more substrate. In such a scenario, the KIE observed in the enzymatic reaction would then exactly match the KIE for the uncatalyzed exchange, which was observed.

A key distinguishing feature of the ketone-binding vs enolbinding models is the kinetic order of the reaction in catalyst. For the ketone-binding model, initial velocities should be directly proportional to catalyst concentration. However, for the enolbinding model, the reaction is fundamentally limited by the speed of the uncatalyzed tautomerization, which would manifest as nonlinearity between catalyst concentration and initial velocity. To examine this hypothesis, we extracted $V_{0}$ values from progress curves with increasing concentrations of TmE8 $(10 \mu \mathrm{M}-150 \mu \mathrm{M}$, Figure 3D). We observed a clear break in the linearity between [TmE8] and $V_{0}$, with the rate of the enzyme-catalyzed reaction asymptotically approaching the 2-fluoroacetophenone exchange rate (Figure 3E). We therefore conclude that TmE8 does not bind the ketone substrate and catalyze tautomerization, but rather reacts directly with the enol tautomer that forms in solution.

The enol-binding mechanism provides clarity to other observations that arose during our studies. The parent enzyme, Tm9D8*, is over 50 -fold slower than the final variant and has a much lower KIE of 3.9 (Figure S4), which we hypothesize is due to the larger steady-state concentration of enol tautomer in solution resulting from slower consumption by the enzyme. Furthermore, if the $K_{M}$ for the substrate were truly $47 \mathrm{mM}$, then $V_{0}$ would be impacted by changes in both $k_{c a t}$ and $K_{\mathrm{M}}$ under the conditions we used to screen for improvements during directed evolution. However, we observed little change in $K_{\mathrm{M}}^{\text {app }}$, despite the significant structural differences between indole and acetophenones. Instead, the enol-binding mechanism suggests the $K_{\mathrm{M}}$ for the true substrate is in the $\mu \mathrm{M}$ regime, thus providing minimal direct selective pressure for this parameter to change. In order to achieve such a low $K_{\mathrm{M}}$, we speculate that the TrpB active site must have exquisite complementarity for the substrate.

To understand the effect of $\mathrm{C} \alpha$ substitutions, we evaluated the substrate scope of the engineered variants and measured the rates of deuterium exchange into those substrates that are compatible with aqueous solutions (Table 1 and SI Section 2.10). Comparing observed rate constants $\left(k_{\text {exg }}\right)$, propiophenone tautomerizes an order of magnitude slower than acetophenone (5.86 $\times 10^{-4} \mathrm{~min}^{-1}$ vs. $4.14 \times 10^{-3} \mathrm{~min}^{-1}$, respectively). However, TmE8 exhibited a higher level of activity with propiophenone than acetophenone, suggesting that small changes in substitutions at the Ca position are distinguished by the enzyme, and positioning of the resulting enol is crucial. In agreement with this hypothesis, TmE8 showed varying degrees of activity with different substituents at Ca. Notably, TmE8 catalyzed the reaction with 2cyanoacetophenone, a substrate electronically similar to 2fluoroacetophenone, with 250 TTN (Figure S9). Intriguingly, the product of this reaction isomerizes to the corresponding enamine, which extends the pi conjugation from the arene to the nitrile, producing an intense absorption band in the visible region (Figure S14). TmE8 showed no activity with 2-phenylacetophenone, suggesting that it does not readily accommodate bulkier substituents at Ca; however, trace activity was observed with benzoylacetone (<1 TTN). We also found that a variant stemming from Pyrococcus furiosus TrpB, PfTrpB ${ }^{7 \mathrm{EE}}$, exhibited similar activity with benzoylacetone (See SI Figure S9). Thus, it appears that multiple TrpB scaffolds are capable of catalyzing this new reaction, broadening the sequence space available to subsequent engineering efforts with other substrates of interest.

As it stands currently, the maximum rate of product formation using TrpB is approximately limited by $k_{\operatorname{exg}}$, but this limitation does not preclude its potential utility. For example, assuming that a $\operatorname{TrpB}$ variant were limited exclusively by $k_{\text {exg, a }}$ reaction with $50 \mathrm{mM}$ 2-fluoroacetophenone would reach completion in less than 4 hours. Under the same conditions, a reaction with acetophenone would near completion in 12 hours. We would also expect the rates of tautomerization, and thus product formation, to be enhanced at higher reaction temperatures, which could be compatible with thermostable variants. Nevertheless, emulating Nature's machinery may comprise the ultimate solution. Despite the fact that the keto-enol equilibrium strongly favors the ketone in aqueous conditions, enolates are common nucleophiles in biology, and several strategies to overcome these unfavorable thermodynamics have evolved naturally. For example, type I aldolases form an intermediate Schiff base between the substrate carbonyl and an active-site lysine residue to effectively reduce the $p K_{a}$ of the $C \alpha$ proton. ${ }^{[21]}$ Although TmE8 does not boast such sophistication, there are no obvious factors that would directly preclude engineering the machinery necessary to effect enolization by an analogous mechanism.

Table 1. Ketone substrate scope of TmE8 and deuterium exchange rate constants $\left(k_{\text {exg }}\right)$.

Entry

[a] 2-cyanoacetophenone is insoluble in buffer. [b] Benzoylacetone hydrolyzes at $55^{\circ} \mathrm{C}$.

In conclusion, we have demonstrated that $\operatorname{TrpB}$ can be evolved for the asymmetric alkylation of ketones and have provided a glimpse into the scope of substrates that may be 
compatible with this new enzymatic transformation. Given that TrpB TmE8 does not influence the keto-enol tautomerization rate, yet exhibits control over the formation of diastereomeric products, we posit that the active site has been engineered to bind a particular configuration of the nucleophilic enol tautomer and direct its approach to the electrophilic amino acrylate. The linear products of the TrpB-catalyzed reactions presented herein spontaneously cyclize to form derivatives of 1-pyrroline-5carboxylic acid, ${ }^{[22]}$ which could be further processed by a suitable imine reductase in a cascade system to furnish stereochemically dense non-canonical prolines. ${ }^{[23]}$ Furthermore, expanding this platform to include aldehydes as nucleophilic substrates and $\beta$ branched amino acids as amino-acrylate precursors would provide access to each carbon substituent on the nitrogen heterocycle. Finally, this study demonstrates that enolates can be intercepted by enzymes, raising the possibility that other classes of enzymes could also be leveraged for asymmetric ketone alkylation in the future.

\section{Acknowledgements}

We thank Nathaniel Goldberg for assistance with NMR interpretation and Dr. Nicholas Porter, Dr. David Miller, and Dr. Benjamin Levin for insightful discussions. This work was supported by the National Institute of General Medical Sciences of the National Institutes of Health under Award Numbers R01GM125887 (to F.H.A.) and DP2-GM137417 (to A.R.B) and the Rothenberg Innovation Initiative at Caltech. N.P.D. was supported by Ruth L. Kirschstein NIH Postdoctoral Fellowship (F32GM131620). The content is solely the responsibility of the authors and does not necessarily represent the official views of the National Institutes of Health.

Keywords: Biocatalysis - Ketones - Nitrogen heterocycles • Directed evolution $\cdot$ Asymmetric catalysis

[1] K. Fesko, M. Gruber-Khadjawi, ChemCatChem 2013, 5, $1248-1272$.

[2] N. G. Schmidt, E. Eger, W. Kroutil, ACS Catal. 2016, 6, $4286-4311$.

[3] R. Cano, A. Zakarian, G. P. McGlacken, Angew. Chemie Int. Ed. 2017, 56, 9278-9290.

[4] E. Watkins-Dulaney, S. Straathof, F. Arnold, ChemBioChem 2021, 22, 5-16.

[5] A. R. Buller, S. Brinkmann-Chen, D. K. Romney, M. Herger, J. Murciano-Calles, F. H. Arnold, Proc. Natl. Acad. Sci. 2015, 112, 14599-14604.
H. Tanaka, K. Tanizawa, T. Arai, K. Saito, T. Arai, K. Soda, FEBS Lett. 1986, 196, 357-360.

[9] J. Murciano-Calles, D. K. Romney, S. Brinkmann-Chen, A. R. Buller, F. H. Arnold, Angew. Chemie - Int. Ed. 2016, 55, 11577-11581.

[10] D. Ferrari, L. H. Yang, E. W. Miles, M. F. Dunn, Biochemistry 2001, 40, 7421-7432.

[11] N. Esaki, K. Soda, Methods Enzymol. 1987, 143, 291-297.

[12] C. E. Boville, D. K. Romney, P. J. Almhjell, M. Sieben, F. H. Arnold, J. Org. Chem. 2018, 83, 7447-7452.

[13] D. K. Romney, J. Murciano-Calles, J. E. Wehrmüller, F. H. Arnold, J. Am. Chem. Soc. 2017, 139, 10769-10776.

[14] E. J. Watkins, P. J. Almhjell, F. H. Arnold, ChemBioChem 2020, 21, 80-83.

[15] D. K. Romney, N. S. Sarai, F. H. Arnold, ACS Catal. 2019, 9, 8726-8730.

[16] M. Dick, N. S. Sarai, M. W. Martynowycz, T. Gonen, F. H. Arnold, J. Am. Chem. Soc. 2019, 141, 19817-19822.

[17] C. J. Welch, X. Gong, W. Schafer, E. C. Pratt, T. Brkovic, Z. Pirzada, J. F. Cuff, B. Kosjek, Tetrahedron Asymmetry 2010, 21, 1674-1681.

[18] H. Zhao, W. Zha, Nat. Protoc. 2006, 1, 1865-1871.

[19] J. R. Keeffe, A. J. Kresge, J. Toullec, Can. J. Chem. 1986, $64,1224-1227$.

P. Haspra, A. Sutter, J. Wirz, Angew. Chemie Int. Ed. English 1979, 18, 617-619.

[21] S. M. Dean, W. A. Greenberg, C. H. Wong, Adv. Synth. Catal. 2007, 349, 1308-1320.

[22] M. J. Bodner, R. Li, R. M. Phelan, M. F. Freeman, K. A. Moshos, E. P. Lloyd, C. A. Townsend, ChemBioChem 2011, 12, 2159-2165.

[23] J. Mangas-Sanchez, S. P. France, S. L. Montgomery, G. A. Aleku, H. Man, M. Sharma, J. I. Ramsden, G. Grogan, N. J. Turner, Curr. Opin. Chem. Biol. 2017, 37, 19-25. 


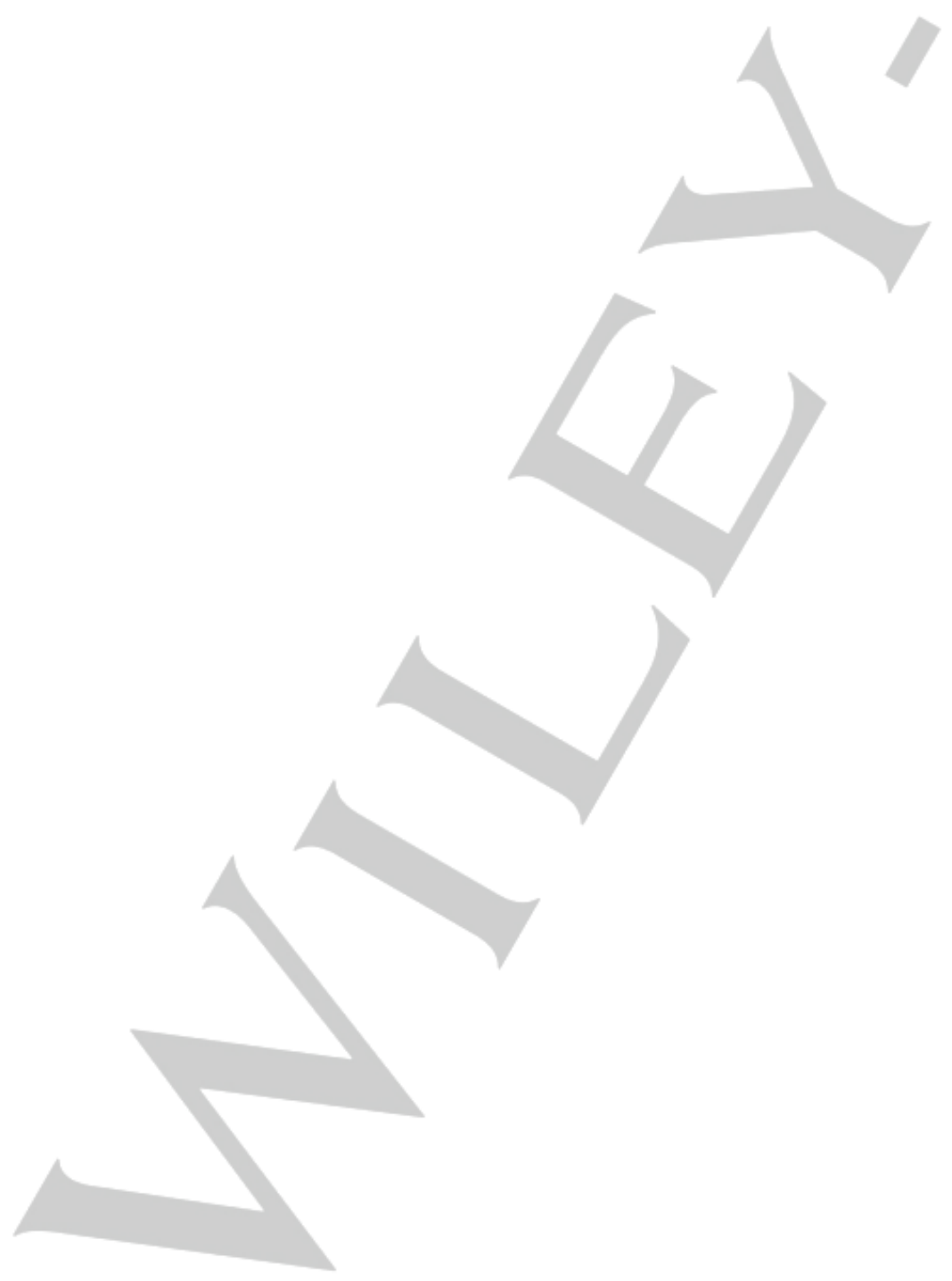


WILEY-VCH

\section{Entry for the Table of Contents}

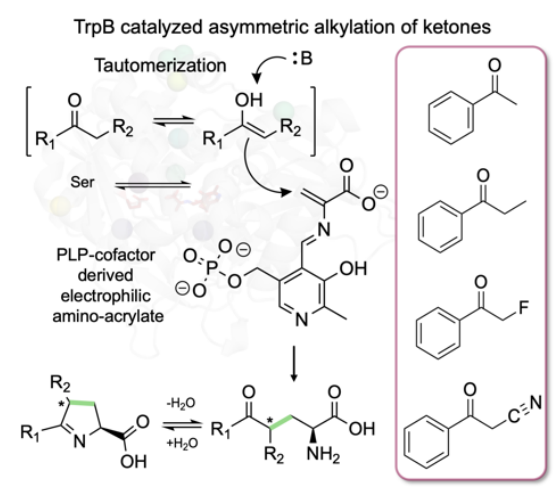

We investigate the capacity of ketone-derived enolates to serve as nucleophiles for tryptophan synthase $\beta$-subunit (TrpB) catalysed reactions. The transient nucleophilic enolate species is intercepted by $\operatorname{TrpB}$, which facilitates selective $\mathrm{C}-\mathrm{C}$ formation, resulting in the asymmetric alkylation of ketones such as propiophenone and 2 -fluoroacetophenone. The products spontaneously cyclize to form highly substituted nitrogen heterocycles that can be reduced to form proline analogs.

Institute and/or researcher Twitter usernames: @francesarnold @Ella_Watkins_@NoahPDunham @Bullerlab 